PROCEEDINGS OF THE

AMERICAN MATHEMATICAL SOCIETY

Volume 138, Number 3, March 2010, Pages 1069-1083

S 0002-9939(09)10170-3

Article electronically published on November 2, 2009

\title{
BEST RESPONSE DYNAMICS FOR CONTINUOUS GAMES
}

\author{
E. N. BARRON, R. GOEBEL, AND R. R. JENSEN
}

(Communicated by Yingfei Yi)

\begin{abstract}
We extend the convergence result of Hofbauer and Sorin for the best response differential inclusions coming from a nonconcave, nonconvex continuous payoff function $U(x, y)$. A counterexample shows that convergence to a Nash equilibrium may not be true if we attempt to generalize the result to a three-person nonzero sum game.
\end{abstract}

\section{INTRODUCTION}

A recent interesting paper by Hofbauer and Sorin [4] considers the best response differential inclusions for a given, continuous function $U: X \times Y \rightarrow \mathbb{R}$, assumed to be concave-convex. Precisely, the best response dynamics they consider is the system

$$
\dot{\xi}(\tau)+\xi(\tau) \in B R_{1}(\eta(\tau)), \quad \dot{\eta}(\tau)+\eta(\tau) \in B R_{2}(\xi(\tau)), \quad \tau \geq 0,
$$

where

$$
B R_{1}(y)=\underset{x \in X}{\arg \max } U(x, y) \text { and } B R_{2}(x)=\underset{y \in Y}{\arg \min } U(x, y) .
$$

This system has been considered by several authors since at least 1991 (see 2], for example). The main result of 4 is that the solution of the best response dynamics converges to a saddle point of the function $U(x, y)$ as $\tau \rightarrow \infty$. This generalized earlier classical work which applied to zero sum matrix games. The practical importance of a convergence result for the best dynamics is the verification that using a best response strategy for each time is ultimately optimal.

The idea of the proof in [4] is to construct the natural function

$$
V(x, y)=\max _{x \in X} U(x, y)-\min _{y \in Y} U(x, y)
$$

and to show that $\tau \rightarrow V(\xi(\tau), \eta(\tau))$ converges to 0 as $\tau \rightarrow \infty$, implying that $V$ is a sort of Lyapunov function for the dynamics. As a consequence, every cluster point of $(\xi(\tau), \eta(\tau))$ must be a saddle of $U$, and there must be cluster points if $X$ and $Y$ are compact.

Received by the editors May 8, 2009, and, in revised form, August 18, 2009.

2010 Mathematics Subject Classification. Primary 91A25, 49J35; Secondary 37B25, 34D20, $26 \mathrm{~B} 25$.

Key words and phrases. Best response, quasiconcave, quasiconvex, Nash equilibrium.

(C)2009 American Mathematical Society 
The goal of this paper is to extend the Hofbauer-Sorin result in a substantial way to nonconcave-nonconvex payoff functions. We will not assume that $U$ is concaveconvex, but merely that the best response sets are convex. This assumption is satisfied not only for concave-convex functions, but, more generally, for quasiconcavequasiconvex functions. Recall that a quasiconvex function $f: A \rightarrow \mathbb{R}$ on a convex set $A \subset \mathbb{R}^{n}$ is a function for which the sublevel sets $\{x \in A \mid f(x) \leq a\}$ are convex for all $a \in \mathbb{R}$. Equivalently, $f(\lambda x+(1-\lambda) y) \leq f(x) \vee f(y), \forall x, y \in A, 0 \leq \lambda \leq 1$. A function $f$ is quasiconcave if $-f$ is quasiconvex.

Just as the extension of the classical von Neumann minimax theorem to the Sion minimax theorem is not straightforward, there are substantial problems encountered in generalizing the Hofbauer-Sorin result. The main problem is that if only quasiconcavity-quasiconvexity is assumed, none of the inequalities used to prove that $V$ decreases along the trajectories are valid. The idea in this paper is to replace $U$ with the concave and convex envelopes of $U$ in each individual variable. It is clear that it is not possible to turn $U$ into a concave-convex function simultaneously. While this idea gives us something to work with, it does not turn out to be enough. The concave and convex envelopes have to be modified so that they contact the original function $U$ only at maximum points (in $x$ ) and minimum points (in $y$ ). This turns out to be a little tricky, but, fortunately, the supremal and infimal convolutions (or Moreau-Yosida convolutions) will satisfy those properties, as we prove in a lemma below.

Starting from $U$ we construct the envelopes $U^{ \pm}$and then $U_{k}^{ \pm}$(the convolutions for fixed $k>0)$, and we work throughout with $U_{k}^{ \pm} \cdot U_{k}^{+}(x, y)$ will be concave in $x$ and $U_{k}^{-}(x, y)$ will be convex in $y$. Our conclusion, however, is that the best response dynamics defined using the functions $U_{k}^{ \pm}$have trajectories which cluster in the set of saddle points for $U$.

Examples exhibited in the second section show that the constructions we use to get convergence are essential. Our first example starts with a discontinuous quasiconcave-quasiconvex payoff function, and we do not use the envelopes or the convolutions. It shows that we may start at a saddle point for $U$, and yet the best response dynamics converge to a point which is not a saddle point. The second example takes a continuous quasiconcave-quasiconvex function $U$ and shows nonconvergence of the best response dynamics to the saddle set.

Finally, in the last section we exhibit an example answering the question as to whether or not a similar convergence result can be obtained for a Nash equilibrium. Our example is a three player nonzero sum game in which the payoff function for each player is actually strictly convex (the players want to minimize their cost). There is a unique Nash equilibrium at $(0,0,0)$. If we construct the best response dynamics we get a system of three linear differential equations. The solution of this system, starting from any nonzero initial point, converges to a cycle. The dynamics do not converge to the Nash equilibrium. Shapley [6] has given an example of a bimatrix game in which the discrete BR dynamics do not converge to a Nash equilibrium.

\section{Convergence to a SAdDle}

We are given a function $U: X \times Y \rightarrow \mathbb{R}$, where $X \subset \mathbb{R}^{n}$ and $Y \subset \mathbb{R}^{m}$ are compact and convex. Assume throughout this paper that

$$
(x, y) \mapsto U(x, y) \text { is continuous on } X \times Y \text {. }
$$


In order to simplify the presentation we will assume the following condition on $U$ :

$$
\begin{aligned}
& x \mapsto U(x, y) \quad \text { is quasiconcave for each fixed } y \in Y, \\
& y \mapsto U(x, y) \quad \text { is quasiconvex for each fixed } x \in X .
\end{aligned}
$$

This assumption can be weakened, as we will see later. Under this assumption it is well known (using the Sion minimax theorem) that there is a saddle value of $U$ in the sense that

$$
\max _{x \in X} \min _{y \in Y} U(x, y)=\min _{y \in Y} \max _{x \in X} U(x, y) .
$$

Remark 2.1. One of the primary goals of the paper 4 is to prove the von Neumann minimax theorem by use of the best response dynamical system. By contrast, we assume conditions sufficient to guarantee the existence of a saddle point for $U$.

Since we are not going to assume any concavity-convexity properties of $U$, we introduce the functions $U^{ \pm}$which will have the convexity properties we need. Define the envelopes on $X \times Y$ by

$$
U^{-}(x, y)=\sup \left\{h^{x}(y) \mid h^{x}(y) \text { is convex in } y \text { for each } x ; h^{x}(y) \leq U(x, y), \forall y \in Y\right\}
$$

and

$U^{+}(x, y)=\inf \left\{g^{y}(x) \mid g^{y}(x)\right.$ is concave in $x$ for each $\left.y ; g^{y}(x) \geq U(x, y), \forall x \in X\right\}$.

$U^{-}(x, \cdot)$ is the greatest convex minorant of $U(x, \cdot)$ as a function of $y$ for each fixed $x$. Similarly, $U^{+}(\cdot, y)$ is the smallest concave majorant of $U(\cdot, y)$ as a function of $x$ for each fixed $y$. We may use the Fenchel conjugates to explicitly construct $U^{ \pm}$by

$$
U^{-}(x, y)=\sup _{p \in \mathbb{R}^{m}}\left\{p \cdot y-U^{*}(x, p)\right\}, \text { where } U^{*}(x, p)=\sup _{y \in Y}\{p \cdot y-U(x, y)\} .
$$

Also,

$$
U^{+}(x, y)=\inf _{p \in \mathbb{R}^{n}}\left\{p \cdot x+U^{\#}(p, y)\right\}, \text { where } U^{\#}(p, y)=\inf _{x \in X}\{p \cdot x+U(x, y)\} .
$$

In addition we will need the following useful characterization. We have that

$$
\begin{aligned}
& U^{-}(x, y)=\min \left\{\sum_{i=1}^{m+1} \lambda_{i} U\left(x, y_{i}\right) \mid \sum_{i=1}^{m+1} \lambda_{i} y_{i}=y, \lambda_{i} \geq 0, \sum \lambda_{i}=1\right\} \\
& U^{+}(x, y)=\max \left\{\sum_{i=1}^{n+1} \mu_{i} U\left(x_{i}, y\right) \mid \sum_{i=1}^{n+1} \mu_{i} x_{i}=x, \mu_{i} \geq 0, \sum \mu_{i}=1\right\}
\end{aligned}
$$

The lower semicontinuity of $y \mapsto U(\cdot, y)$ guarantees that $U^{-}(x, \cdot)=U^{* *}(x, \cdot)$, and the upper semicontinuity of $x \mapsto U(x, \cdot)$ guarantees the equality $U^{+}(\cdot, y)=$ $U^{\# \#}(\cdot, y)$. The next lemma gives some basic properties of $U^{ \pm}$.

Lemma 2.2. We have

(1) $U^{-}(x, y) \leq U(x, y) \leq U^{+}(x, y), \forall x \in X, y \in Y$.

(2) The function $(x, y) \mapsto U^{-}(x, y)$ is lower semicontinuous in $(x, y) \in X \times Y$, convex in $y \in Y$ for any fixed $x \in X$, and continuous in $x \in X$ for any fixed $y \in Y$. The function $(x, y) \mapsto U^{+}(x, y)$ is upper semicontinuous in $(x, y) \in X \times Y$, concave in $x \in X$ for any fixed $y \in Y$, and continuous in $y \in Y$ for any fixed $x \in X$.

(3) If $U(x, y)$ is quasiconcave in $x$, then $U^{-}(x, y)$ is also quasiconcave in $x$ for each fixed $y \in Y$. Similarly, if $U(x, y)$ is quasiconvex in $y$, then $U^{+}(x, y)$ is also quasiconvex in $y$ for each fixed $x$. 
Proof. Part (1) is obvious by construction.

For part (2), lower semicontinuity of $U^{-}(x, y)$ in $(x, y) \in X \times Y$ comes from the fact that parametric minimization preserves that property; see, for example, [7, Theorem 1.17]. Convexity of $U^{-}(x, y)$ in $y \in Y$ comes directly from the definition of $U^{-}$. Upper semicontinuity, and hence continuity, of $U^{-}(x, y)$ in $x$ for a fixed $y$ can be shown using the fact that the minimum defining $U^{-}(x, y)$ in $(2.2)$ is attained. Indeed, if $U^{-}(x, y)=\sum_{i=1}^{m+1} \lambda_{i} U\left(x, y_{i}\right)$ for appropriate $\lambda_{i}$ 's and $y_{i}$ 's, then, by definition, $U^{-}\left(x^{\prime}, y\right) \leq \sum_{i=1}^{m+1} \lambda_{i} U\left(x^{\prime}, y_{i}\right)$ and thus

$$
\limsup _{x^{\prime} \rightarrow x} U^{-}\left(x^{\prime}, y\right) \leq \sum_{i=1}^{m+1} \lambda_{i} \lim _{x^{\prime} \rightarrow x} U\left(x^{\prime}, y_{i}\right)=U^{-}(x, y) .
$$

Properties of $U^{+}$follow by symmetry.

(3) We have the conjugate $U^{*}(x, p)=\sup _{y \in Y}\{p \cdot y-U(x, y)\}$. Let $x_{1}, x_{2} \in$ $X, 0<\lambda<1$, and set $x_{\lambda}=\lambda x_{1}+(1-\lambda) x_{2} \in X$. Then, since $x \mapsto U(x, y)$ is quasiconcave,

$$
\begin{aligned}
U^{*}\left(x_{\lambda}, p\right) & =\sup _{y \in Y}\left\{p \cdot y-U\left(x_{\lambda}, y\right)\right\} \\
& \leq \sup _{y \in Y}\left\{p \cdot y-\left(U\left(x_{1}, y\right) \wedge U\left(x_{2}, y\right)\right)\right\} \\
& =\sup _{y \in Y}\left\{\left(p \cdot y-U\left(x_{1}, y\right)\right) \vee(p \cdot y-U(x, y))\right\} \\
& =U^{*}\left(x_{1}, p\right) \vee U^{*}\left(x_{2}, p\right) .
\end{aligned}
$$

Therefore $x \mapsto U^{*}(x, p)$ is quasiconvex. In a similar way, it will then follow that $x \mapsto U^{-}(x, y)$ is quasiconcave.

The next lemma tells us that the convex envelope of a lower semicontinuous function has the same minimum as the original function and that the concave envelope of an upper semicontinuous function has the same maximum.

Lemma 2.3. For any lower semicontinuous function $f: Y \rightarrow \mathbb{R}$ we have

$$
\inf \{f(y) \mid y \in Y\}=\inf \left\{f^{* *}(y) \mid y \in Y\right\},
$$

where $f^{* *}$ is the greatest convex minorant of $f$ (the second Fenchel conjugate). For any upper semicontinuous function $f: X \rightarrow \mathbb{R}$ we have

$$
\sup \{f(x) \mid x \in X\}=\sup \left\{f^{\# \#}(x) \mid x \in X\right\},
$$

where $f^{\# \#}$ is the smallest concave majorant of $f$.

Proof. We only prove the first statement. Under our assumptions $f$ is bounded from below, so all the infima are finite. Since $f^{* *}(y) \leq f(y)$ for all $y \in Y$, we have $\inf \{f(y) \mid y \in Y\} \geq \inf \left\{f^{* *}(y) \mid y \in Y\right\}$. Now let $\inf _{y} f(y)=\alpha$. Then

$$
\begin{aligned}
f^{* *}(y)=\min \left\{\sum_{i=1}^{n+1} \lambda_{i} f\left(y_{i}\right) \mid \sum_{i=1}^{n+1} \lambda_{i} y_{i}=y, \lambda_{i} \geq 0, \sum \lambda_{i}=1\right\} \\
\quad \geq \min \left\{\sum_{i=1}^{n+1} \lambda_{i} \alpha \mid \sum_{i=1}^{n+1} \lambda_{i} y_{i}=x, \lambda_{i} \geq 0, \sum \lambda_{i}=1\right\}=\alpha .
\end{aligned}
$$

Consequently, $\inf \{f(y) \mid y \in Y\} \leq \inf \left\{f^{* *}(y) \mid y \in Y\right\}$. 
The previous lemma allows us to conclude that $y \mapsto U^{-}(x, y)$ has the same minimum as $y \mapsto U(x, y)$ and that $x \mapsto U^{+}(x, y)$ has the same maximum as $x \mapsto$ $U(x, y)$. Unfortunately, it is possible that $U^{-}(x, y)=U(x, y)$ at some point $(x, y) \in$ $X \times Y$, and yet the $y$-component of this point is not a minimizer of $U(x, y)$, for $x$ fixed. That is, the convex envelope of $U$ in $y$ can contact $U$ at points other than minimizers of $U$. The amazing thing is that if we take the inf convolution (also known as the Moreau convolution) of $U^{-}$in the $y$ variable, the resulting function will still be convex but will only contact $U^{-}$on the minimum set of $U^{-}$, and that coincides with the minimum set of $U$. A similar statement holds for $U^{+}$.

To recall the definition and properties of the Moreau convolutions we define for each $k>0$, each $x \in X$, and each $y \in \mathbb{R}^{m}$ the inf convolution

$$
U_{k}^{-}(x, y)=\inf \left\{U^{-}(x, z)+\frac{k}{2}|z-y|^{2}: z \in Y\right\} .
$$

Similarly, for each $x \in \mathbb{R}^{n}$ and each $y \in Y$, the sup convolution is given by

$$
U_{k}^{+}(x, y)=\sup \left\{U^{+}(z, y)-\frac{k}{2}|z-x|^{2}: z \in X\right\} .
$$

We will fix $k>0$ throughout this paper. Properties of the inf and sup convolutions are well known, and we summarize their main points in the following lemma. Apart from the convexity of $U_{k}^{-}(x, \cdot)$ and concavity of $U^{+}(\cdot, y)$, properties (8) and (9) are the main properties we need.

Lemma 2.4. Define the functions $f_{k}(y)=U_{k}^{-}(x, y)$ for each fixed $x \in X$, and $g_{k}(x)=U_{k}^{+}(x, y)$. We have the properties:

(1) $f_{k}(y)=U_{k}^{-}(x, y)$ is convex and $g_{k}(x)=U_{k}^{+}(x, y)$ is concave.

(2) $f_{k}: Y \rightarrow \mathbb{R}$ and $g_{k}: X \rightarrow \mathbb{R}$ are continuously differentiable.

(3) Set $M_{k}(y)=\arg \min _{z \in Y} U^{-}(x, z)+\frac{k}{2}|z-y|^{2} . M_{k}(y)$ is single valued and continuous, and $D f_{k}(y)=\left(y-z_{k}\right) k$, where $z_{k}=M_{k}(y)$.

(4) Set $N_{k}(x)=\arg \max _{z \in X} U^{+}(z, y)-\frac{k}{2}|z-x|^{2} . N_{k}(x)$ is single valued and continuous, and $D g_{k}(x)=\left(x-z_{k}\right) k$, where $z_{k}=N_{k}(x)$.

(5) The functions $U_{k}^{-}$and $U_{k}^{+}$are continuous in $(x, y) \in X \times Y$.

(6) $\arg \min _{y \in Y} U_{k}^{-}(x, y)=\arg \min _{y \in Y} U^{-}(x, y)$ for each fixed $x \in X$.

(7) $\arg \max _{x \in X} U_{k}^{+}(x, y)=\arg \max _{x \in X} U^{+}(x, y)$ for each fixed $y \in Y$.

(8) If $U^{-}(x, y)=U_{k}^{-}(x, y)$ for some $y \in Y$, then $y \in \arg \min _{y} U^{-}(x, y)$.

(9) If $U^{+}(x, y)=U_{k}^{+}(x, y)$ for some $x \in X$, then $x \in \arg \max _{x} U^{+}(x, y)$.

Proof. Properties (1)-(4) are well known (see [7, pp. 19-21, Theorem 2.26], for example).

For property (5), the function $(x, y) \mapsto U_{k}^{-}(x, y)$ is lower semicontinuous on $X \times Y$ since parametric optimization preserves lower semicontinuity; see [7, Theorem 1.17]. For each $y \in Y, x \mapsto U_{k}^{-}(x, y)$ is upper semicontinuous, and hence continuous, in $x \in X$, which can be shown using the fact that the infimum defining $U_{k}^{-}(x, y)$ is attained. The functions $y \mapsto U_{k}^{-}(x, y)$ are Lipschitz continuous uniformly in $x \in X$. This follows from property (3). Now, continuity of $U_{k}^{-}$in $(x, y)$ is immediate from the continuity in $x$ and the equicontinuity in $y$. Continuity of $U_{k}^{+}$follows in a similar way.

For property (6), observe that

$$
\inf _{y} U_{k}^{-}(x, y)=\inf _{z} \inf _{y}\left[U^{-}(x, z)+\frac{k}{2}|y-z|^{2}\right]=\inf _{z} U^{-}(x, z),
$$


and if $y_{0} \in \arg \min _{y} U^{-}(x, y)$, then

$$
U_{k}^{-}\left(x, y_{0}\right) \geq \inf _{y} U_{k}^{-}(x, y)=\inf _{z} U^{-}(x, z)=U^{-}\left(x, y_{0}\right) \geq U_{k}^{-}\left(x, y_{0}\right),
$$

implying that $\arg \min _{y} U^{-}(x, y) \subset \arg \min _{y} U_{k}^{-}(x, y)$. Conversely, if we take a point $y_{k} \in \arg \min _{y} U_{k}^{-}(x, y)$, then there is $z_{k} \in Y$ such that

$$
U_{k}^{-}\left(x, y_{k}\right)=U^{-}\left(x, z_{k}\right)+\frac{k}{2}\left|z_{k}-y_{k}\right|^{2} .
$$

If $z_{k} \neq y_{k}$, then

$$
U_{k}^{-}\left(x, y_{k}\right)>U^{-}\left(x, z_{k}\right) \geq \inf _{y} U^{-}(x, y)=\inf _{y} U_{k}^{-}(x, y)=U_{k}^{-}\left(x, y_{k}\right) .
$$

This contradiction gives $U_{k}^{-}\left(x, y_{k}\right)=U^{-}\left(x, y_{k}\right)$ and then $y_{k} \in \arg \min _{y} U^{-}(x, y)$, completing the proof of $(6)$.

To see (8), we will show that for a convex lower semicontinuous function $f$ : $X \rightarrow \mathbb{R}$, the inf convolution of $f, f_{k}(x)=\inf _{y}\left\{f(y)+\frac{k}{2}|y-x|^{2}\right\}$, satisfies the property that if $x_{0}$ is a point of contact $f_{k}\left(x_{0}\right)=f\left(x_{0}\right)$, then $x_{0} \in \arg \min _{x} f$. It is straightforward to calculate

$$
f_{k}(x)=\sup _{p}\left\{p \cdot x-f^{*}(p)-\frac{1}{2 k}|p|^{2}\right\},
$$

where $f^{*}(p)$ is the conjugate of $f$. We have $f_{k}^{*}(p)=f^{*}(p)+\frac{1}{2 k}|p|^{2}$.

Now we use the following fact: If $f$ and $g$ are convex functions satisfying $f(x) \geq$ $g(x), f\left(x_{0}\right)=g\left(x_{0}\right)$ at some $x_{0}$, then $\partial g\left(x_{0}\right) \subset \partial f\left(x_{0}\right)$. Indeed, if $q \in \partial g\left(x_{0}\right)$, then $g\left(x_{0}\right)+g^{*}(q)=q \cdot x_{0}$ and, therefore,

$$
q \cdot x_{0}=g\left(x_{0}\right)+g^{*}(q) \geq f\left(x_{0}\right)+f^{*}(q) \text {, since } f \geq g \Longrightarrow f^{*} \leq g^{*} .
$$

But then $q \cdot x_{0}-f^{*}(q) \geq f\left(x_{0}\right)=\sup _{p}\left(p \cdot x_{0}-f^{*}(p)\right)$, implying that $q \in \partial f\left(x_{0}\right)$.

Suppose now that $f_{k}\left(x_{0}\right)=f\left(x_{0}\right)$ and suppose $p_{0}$ is a subgradient of $f_{k}$ at $x_{0}$. Then, since $f_{k} \leq f, p_{0}$ is also a subgradient of $f$ at $x_{0}$ and

$$
p_{0} \cdot x_{0}=f_{k}\left(x_{0}\right)+f_{k}^{*}\left(p_{0}\right)=f\left(x_{0}\right)+f^{*}\left(p_{0}\right)+\frac{1}{2 k}\left|p_{0}\right|^{2}=p_{0} \cdot x_{0}+\frac{1}{2 k}\left|p_{0}\right|^{2} .
$$

This implies that $p_{0}=0$. Consequently, $x_{0}$ must be a minimizer of $f$.

A proof of (9) which requires no convex analysis proceeds as follows. Let $f$ : $Y \rightarrow \mathbb{R}$ be a concave function and suppose that

$$
f^{k}(y)=\sup _{u \in Y}\left\{f(u)-\frac{k}{2}|u-y|^{2}\right\}=f(y), \text { for some } y \in Y,
$$

but $y$ is not a maximum of $f$. Then, $f(y)<f(z)$ for some $z \in Y$ and

$$
\begin{aligned}
f^{k}(y) & =\sup _{u \in Y}\left\{f(u)-\frac{k}{2}|u-y|^{2}\right\} \\
& \geq \sup _{\lambda \in[0,1]}\left\{f((1-\lambda) y+\lambda z)-\frac{k}{2}|(1-\lambda) y+\lambda z-y|^{2}\right\} \\
& \geq \sup _{\lambda \in[0,1]}\left\{(1-\lambda) f(y)+\lambda f(z)-\frac{k \lambda^{2}}{2}|z-y|^{2}\right\} \\
& =f(y)+\sup _{\lambda \in[0,1]}\left\{\lambda(f(z)-f(y))-\frac{k \lambda^{2}}{2}|z-y|^{2}\right\} \\
& >f(y),
\end{aligned}
$$


where the last strict inequality follows from $f(z)-f(y)>0$. This is a contradiction. Thus, if $f^{k}(y)=f(y)$ for some $y \in Y$, then $y$ is a maximum of $f$.

Notice that

$$
U_{k}^{-}(x, y) \leq U^{-}(x, y) \leq U(x, y) \leq U^{+}(x, y) \leq U_{k}^{+}(x, y) .
$$

Consequently, whenever $U_{k}^{-}(x, y)=U_{k}^{+}(x, y)$ we have equality throughout.

Throughout the rest of this paper we fix $k>0$. Now we set

$$
V(x, y)=\max _{\xi} U_{k}^{-}(\xi, y)-\min _{\eta} U_{k}^{+}(x, \eta) .
$$

Since $y \mapsto U_{k}^{-}(x, y)$ is convex and $x \mapsto U_{k}^{+}(x, y)$ is concave, it is easy to see that $V: X \times Y \rightarrow \mathbb{R}$ is jointly convex in $(x, y)$.

We define $X(U) \times Y(U)$ as the set of saddle points of $U$. This notation is justified by the following remark.

Remark 2.5. When $U(x, y)$ is quasiconcave-quasiconvex on $X \times Y$, the set of saddle points is a Cartesian product of convex sets. This follows from the following facts.

For any two saddle points, $\left(x_{1}, y_{1}\right),\left(x_{2}, y_{2}\right) \in X \times Y$,

$$
U\left(x_{1}, y_{2}\right) \geq U\left(x_{1}, y_{1}\right) \geq U\left(x_{2}, y_{1}\right) \geq U\left(x_{2}, y_{2}\right) \geq U\left(x_{1}, y_{2}\right),
$$

so all are equal. Hence $U\left(x_{1}, y_{1}\right)=U\left(x_{2}, y_{2}\right)=U\left(x_{1}, y_{2}\right)=U\left(x_{2}, y_{1}\right)=s$, where $s$ is the saddle value.

Quasiconcavity, invoked with $y_{1}$ and then $y_{2}$ fixed, together with the fact that $\left(x_{1}, y_{1}\right)$ and $\left(x_{2}, y_{2}\right)$ are saddle points, implies $U\left(\left[x_{1}, x_{2}\right], y_{i}\right)=s$ for $i=1,2$, where $\left[x_{1}, x_{2}\right]$, respectively $\left(x_{1}, x_{2}\right)$, denotes the closed, respectively open, line segment joining $x_{1}, x_{2}$. Quasiconvexity, invoked for each $y$ in $\left(y_{1}, y_{2}\right)$, then implies $U\left(\left(x_{1}, x_{2}\right), y\right) \leq s$ for $y \in\left(y_{1}, y_{2}\right)$. So $U\left(\left(x_{1}, x_{2}\right),\left(y_{1}, y_{2}\right)\right) \leq s$.

Symmetry shows that $U\left(\left(x_{1}, x_{2}\right),\left(y_{1}, y_{2}\right)\right) \geq s$. Hence $U=s$ on $\left[x_{1}, x_{2}\right] \times\left[y_{1}, y_{2}\right]$. This shows that the set of saddle points is a product set.

Lemma 2.6. If $U$ has a saddle value, i.e. (2.1) holds, then $V(x, y) \geq 0, \forall(x, y) \in$ $X \times Y$.

Of course, if $U$ is quasiconcave-quasiconvex, then (2.1) holds. It is worth noting that this is the only place in which we need to assume that (2.1) holds. If it was automatically true or if somehow it could be deduced that $V \geq 0$ as it is in the concave-convex case, then we would actually prove the existence of a saddle point, exactly as in [4].

Proof. We have from (2.1), (2.5), and Lemma 2.4

$$
\begin{aligned}
& \text { (2.6) } \max _{x} U_{k}^{-}(x, y) \geq \min _{y} \max _{x} U_{k}^{-}(x, y) \geq \max _{x} \min _{y} U_{k}^{-}(x, y)=\max _{x} \min _{y} U(x, y) \\
& =\min _{y} \max _{x} U(x, y)=\min _{y} \max _{x} U_{k}^{+}(x, y) \geq \max _{x} \min _{y} U_{k}^{+}(x, y) \geq \min _{y} U_{k}^{+}(x, y) .
\end{aligned}
$$

Now we consider the best response dynamics. We need to introduce the best response sets.

Definition 2.7. For $(x, y) \in X \times Y$, set

$$
B R_{1}^{-}(y)=\arg \max _{\xi \in X} U_{k}^{-}(\xi, y) \text { and } B R_{2}^{+}(x)=\arg \min _{\eta \in Y} U_{k}^{+}(x, \eta) \text {. }
$$

$B R_{1}^{-}(y)$ is the set of best responses of player 1 to $y$, and $B R_{2}^{+}(x)$ is the set of best responses of player 2 to $x$. 
Lemma 2.8. Assuming $U: X \times Y \rightarrow \mathbb{R}$ is continuous and quasiconcave-quasiconvex, $B R_{1}^{-}: Y \rightarrow X$ and $B R_{2}^{+}: X \rightarrow Y$ are both upper semicontinuous set valued maps with values which are nonempty and compact convex subsets of $X$ and $Y$, respectively.

Proof. This is Theorem 1.17 in [7, so we only indicate the proof for $B R_{2}^{+}$.

First observe that from property (5) of Lemma 2.4. $U_{k}^{-}(x, y)$ is jointly continuous in $(x, y) \in X \times Y$.

Let $y_{n} \in B R_{2}^{+}\left(x_{n}\right)$ and assume that $X \ni x_{n} \rightarrow \bar{x} \in X$ and $Y \ni y_{n} \rightarrow \bar{y} \in Y$. We must show $\bar{y} \in B R_{2}^{+}(\bar{x})$. Let $\alpha>\inf _{y} U_{k}^{+}(\bar{x}, y)$. Continuity of $U_{k}^{-}$tells us that for large enough $n, \alpha>\inf _{y} U_{k}^{+}\left(x_{n}, y\right)=U_{k}^{+}\left(x_{n}, y_{n}\right)$. This implies that $\alpha>U_{k}^{+}(\bar{x}, \bar{y})$, and, since $\alpha$ was arbitrary, $\inf _{y} U_{k}^{+}(\bar{x}, y) \geq U_{k}^{+}(\bar{x}, \bar{y})$. This says $\bar{y} \in B R_{2}^{+}(\bar{x})$.

Here is the best response dynamics. Fix a point $(x, y) \in X \times Y$. Define $\xi(0)=$ $x, \eta(0)=y$, and for $\tau>0$,

$$
\begin{aligned}
& \dot{\xi}(\tau)+\xi(\tau)=\alpha(\tau) \quad \in B R_{1}^{-}(\eta(\tau)), \\
& \dot{\eta}(\tau)+\eta(\tau)=\beta(\tau) \quad \in B R_{2}^{+}(\xi(\tau)) .
\end{aligned}
$$

Translated, this means that for all $\tau \geq 0$,

$$
\max _{x \in X} U_{k}^{-}(x, \eta(\tau))=U_{k}^{-}(\alpha(\tau), \eta(\tau)) \text { and } \min _{y \in Y} U_{k}^{+}(\xi(\tau), y)=U_{k}^{+}(\xi(\tau), \beta(\tau)) .
$$

Observe that $\alpha$ depends on $\eta$ and $\beta$ depends on $\xi$ so that the differential inclusions form a coupled system. Standard results in differential inclusions ([5], Theorem 3, p. 98]) give us the existence of an absolutely continuous solution $(\xi(\cdot), \eta(\cdot))$ for all time $\tau \geq 0$.

Lemma 2.9. Consider $v(\tau)=V(\xi(\tau), \eta(\tau)), \tau \geq 0$. Then for almost every $\tau>0$,

$$
\frac{d v}{d \tau}=D_{y} U_{k}^{-}(\alpha(\tau), \eta(\tau))(\beta(\tau)-\eta(\tau))-D_{x} U_{k}^{+}(\xi(\tau), \beta(\tau))(\alpha(\tau)-\xi(\tau))
$$

Proof. Since precise details for the concave-convex case are given in [4, we give only a sketch of the proof. We have from [7, Theorem 10.13, p. 433] that

$$
\begin{aligned}
\frac{d V(\xi(\tau), \eta(\tau))}{d \tau}= & \max \left\{D_{y} U_{k}^{-}(x, \eta(\tau)) \dot{\eta}(\tau) \mid x \in \arg \max _{x} U_{k}^{-}(x, \eta(\tau))\right\} \\
& -\min \left\{D_{x} U_{k}^{+}(\xi(\tau), y) \dot{\xi}(\tau) \mid y \in \arg \min _{y} U_{k}^{+}(\xi(\tau), y)\right\} \\
= & D_{y} U_{k}^{-}(\alpha(\tau), \eta(\tau)) \dot{\eta}(\tau)-D_{x} U_{k}^{+}(\xi(\tau), \beta(\tau)) \dot{\xi}(\tau) \\
= & D_{y} U_{k}^{-}(\alpha(\tau), \eta(\tau))(\beta(\tau)-\eta(\tau)) \\
& -D_{x} U_{k}^{+}(\xi(\tau), \beta(\tau))(\alpha(\tau)-\xi(\tau)) .
\end{aligned}
$$

Using the lemma we compute using the convexity of $y \mapsto U_{k}^{-}(\cdot, y)$ and concavity of $x \mapsto U_{k}^{+}(x, \cdot)$,

$$
\begin{aligned}
\frac{d v}{d \tau} & =D_{y} U_{k}^{-}(\alpha(\tau), \eta(\tau))(\beta(\tau)-\eta(\tau))-D_{x} U_{k}^{+}(\xi(\tau), \beta(\tau))(\alpha(\tau)-\xi(\tau)) \\
& \leq U_{k}^{-}(\alpha, \beta)-U_{k}^{-}(\alpha, \eta)-\left(U_{k}^{+}(\alpha, \beta)-U_{k}^{+}(\xi, \beta)\right) \\
& =-V(\xi, \eta)-\left(U_{k}^{+}(\alpha, \beta)-U_{k}^{-}(\alpha, \beta)\right) \\
& =-v(\tau)-\left(U_{k}^{+}(\alpha(\tau), \beta(\tau))-U_{k}^{-}(\alpha(\tau), \beta(\tau))\right) .
\end{aligned}
$$


Remark 2.10. At this point we have the desired inequality for $v(\tau)$, except that we have the additional term $U_{k}^{+}-U_{k}^{-}$. Without this term we could conclude $v(\tau) \rightarrow 0$ as $\tau \rightarrow \infty$ and then conclude $\max _{x} U_{k}^{-}\left(x, y^{*}\right)=\min _{y} U_{k}^{+}\left(x^{*}, y\right)$ for some $\left(x^{*}, y^{*}\right) \in X \times Y$. That does not necessarily give a saddle point of $U$. The additional term $U_{k}^{+}-U_{k}^{-}$will eventually be used to give us what we need.

Define

$$
F((x, \dot{x}),(y, \dot{y}))=U_{k}^{+}(\dot{x}+x, \dot{y}+y)-U_{k}^{-}(\dot{x}+x, \dot{y}+y) .
$$

Set $h(\tau)=U_{k}^{+}(\alpha(\tau), \beta(\tau))-U_{k}^{-}(\alpha(\tau), \beta(\tau))=F((\xi(\tau), \dot{\xi}(\tau)),(\eta(\tau), \dot{\eta}(\tau)))$. We know that $h(\tau) \geq 0, \forall \tau \geq 0$.

At this point we have shown that

$$
\frac{d v}{d \tau} \leq-v(\tau)-h(\tau), \quad \tau \geq 0
$$

Equivalently, for any $0 \leq s<t$

$$
V(\xi(t), \eta(t)) e^{t}-V(\xi(s), \eta(s)) e^{s}+\int_{s}^{t} h(r) e^{r} d r \leq 0 .
$$

That is,

$$
V(\xi(t), \eta(t)) e^{t}-V(\xi(s), \eta(s)) e^{s}+\int_{s}^{t} F((\xi(r), \dot{\xi}(r)),(\eta(r), \dot{\eta}(r))) e^{r} d r \leq 0 .
$$

This says that $(\xi(\cdot), \eta(\cdot))$ is a monotone trajectory with respect to the functions $(V, F)$ of the differential inclusion

$$
(\dot{\xi}, \dot{\eta}) \in\left(B R_{1}^{-}(\eta)-\xi, B R_{2}^{+}(\xi)-\eta\right) .
$$

Therefore, using [5. Theorem 3, p. 311], we conclude that $(\xi(\tau), \dot{\xi}(\tau)),(\eta(\tau), \dot{\eta}(\tau))$ have almost cluster points $\left(x^{*}, v^{*}\right),\left(y^{*}, w^{*}\right)$, respectively, as $\tau \rightarrow \infty$, and

$$
\alpha^{*}:=v^{*}+x^{*} \in B R_{1}^{-}\left(y^{*}\right) \text { and } \beta^{*}:=w^{*}+y^{*} \in B R_{2}^{+}\left(x^{*}\right) .
$$

In addition,

$$
F\left(\left(x^{*}, v^{*}\right),\left(y^{*}, w^{*}\right)\right)=U_{k}^{+}\left(\alpha^{*}, \beta^{*}\right)-U_{k}^{-}\left(\alpha^{*}, \beta^{*}\right)=0 .
$$

For the reader's convenience we recall that $x^{*}$ is an almost cluster point of $\xi(\tau)$ when $\tau \rightarrow \infty$ if for any $\varepsilon>0$, the set $\left\{\tau \in[0, \infty)|| \xi(\tau)-x^{*} \mid \leq \varepsilon\right\}$ has infinite Lebesgue measure.

Now we combine the facts $U_{k}^{+}\left(\alpha^{*}, \beta^{*}\right)=U_{k}^{-}\left(\alpha^{*}, \beta^{*}\right)$, inequalities (2.5), and Lemma 2.4(8) and (9) to conclude that

$$
U\left(\alpha^{*}, \beta^{*}\right)=\min _{y \in Y} U\left(\alpha^{*}, y\right)=\max _{x \in X} U\left(x, \beta^{*}\right),
$$

and so $\left(\alpha^{*}, \beta^{*}\right)$ is a saddle point for $U$.

We have established the following theorem.

Theorem 2.11. Assume that $B R_{1}^{-}: Y \rightarrow X$ and $B R_{2}^{+}: X \rightarrow Y$ are convex upper semicontinuous correspondences. Suppose also that $U: X \times Y \rightarrow \mathbb{R}$ has a saddle value; i.e., (2.1) holds. Any almost cluster point of the best response dynamics $(\alpha(\cdot)=\xi(\cdot)+\dot{\xi}(\cdot), \beta(\cdot)=\eta(\cdot)+\dot{\eta}(\cdot))$ with initial point $(x, y) \in X \times Y$ is a saddle point in $X(U) \times Y(U)$. 
We still have to prove that the actual trajectories of the best response dynamics have almost cluster points which are actually saddle points and not just $(\alpha, \beta)$. To see why this is true, let $\varepsilon>0$ and let $T_{\varepsilon}$ be a set of infinite Lebesgue measure such that for $\tau \in T_{\varepsilon}, \alpha(\tau) \in X(U)+\varepsilon B$, where $B$ is the closed until ball. We know that such $T_{\varepsilon}$ exists because of Theorem 2.11. For simplicity, denote $X_{\varepsilon}=X(U)+\varepsilon B$. We define

$$
\Phi(\xi(\tau))=\frac{1}{2}\left|\xi(\tau)-\pi_{X_{\varepsilon}}(\xi(\tau))\right|^{2},
$$

where $\pi_{X_{\varepsilon}}(x)$ denotes the projection of $x$ onto the closed convex set $X_{\varepsilon}$. We have, for $\tau \in T_{\varepsilon}$,

$$
\begin{aligned}
\frac{d \Phi}{d \tau} & =\left\langle\xi(\tau)-\pi_{X_{\varepsilon}}(\xi(\tau)), \dot{\xi}(\tau)\right\rangle \\
& =\left\langle\xi(\tau)-\pi_{X_{\varepsilon}}(\xi(\tau)), \alpha(\tau)-\xi(\tau)\right\rangle \\
& =\left\langle\xi(\tau)-\pi_{X_{\varepsilon}}(\xi(\tau)), \alpha(\tau)-\pi_{X_{\varepsilon}}(\xi(\tau))+\pi_{X_{\varepsilon}}(\xi(\tau))-\xi(\tau)\right\rangle \\
& =-\left|\xi(\tau)-\pi_{X_{\varepsilon}}(\xi(\tau))\right|^{2}+\left\langle\xi(\tau)-\pi_{X_{\varepsilon}}(\xi(\tau)), \alpha(\tau)-\pi_{X_{\varepsilon}}(\xi(\tau))\right\rangle \\
& \leq-\Phi(\xi(\tau)) .
\end{aligned}
$$

The last inequality follows from the fact that

$$
\left\langle\xi(\tau)-\pi_{X_{\varepsilon}}(\xi(\tau)), \alpha(\tau)-\pi_{X_{\varepsilon}}(\xi(\tau))\right\rangle \leq 0,
$$

since $\alpha(\tau) \in X_{\varepsilon}$ for $\tau \in T_{\varepsilon}$ (by Theorem 2.11), $\xi(\tau)-\pi_{X_{\varepsilon}}(\xi(\tau))$ is a (proximal) normal to $X_{\varepsilon}$ at $\pi_{X_{\varepsilon}}(\xi(\tau))$, and by the elementary fact in [1, Prop. 1.10]. We conclude from $d \Phi / d \tau \leq-\Phi(\xi(\tau))$ that $\Phi(\xi(\tau)) \rightarrow 0$ as $\tau \rightarrow \infty$, at least along a sequence of times, and hence $x^{*} \in X(u)+\varepsilon B$, for any $\varepsilon>0$. Consequently $x^{*} \in X(U)$.

A similar argument shows that $y^{*} \in Y(U)$. The following theorem has been proved.

Theorem 2.12. Assume that $B R_{1}^{-}: Y \rightarrow X$ and $B R_{2}^{+}: X \rightarrow Y$ are convex upper semicontinuous correspondences. Suppose also that $U: X \times Y \rightarrow \mathbb{R}$ has a saddle value; i.e., (2.1) holds. Any almost cluster point of the trajectories of the best response dynamics $(\xi(\cdot), \eta(\cdot))$ with initial point $(x, y) \in X \times Y$ is an element of the saddle set $X(U) \times Y(U)$.

Remark 2.13. The assumptions of continuity and quasiconcavity-quasiconvexity of $U$ can be weakened. In fact, what was really used in the proofs of Theorem 2.11 and Theorem 2.12 is the following:

- the best response mappings $B R_{1}^{-}: Y \rightarrow X$ and $B R_{2}^{+}: X \rightarrow Y$ are upper semicontinuous and have nonempty, closed, and convex values;

- the function $U$ has a saddle value on $X \times Y$.

The two conditions of course hold when $U$ is continuous and quasiconcave in $x$ and quasiconvex in $y$, but they may hold in other cases as well.

\section{ExAmples}

Example 1. Our first example exhibits a quasiconcave-quasiconvex function for which the best response dynamics, using only the function $U(x, y)$ and not the convex envelopes, do not work. In fact, there is a solution that starts at a saddle point of $U$ and converges to a point that is not a saddle point. The augmented dynamics, based on the concave and convex hulls, fix this issue, i.e., give convergence to the 
saddle set. This shows that even though a quasiconcave - quasiconvex function is known to have a saddle point, the best response dynamics will not necessarily converge to it.

Let $U:[0,1] \times[0,1] \rightarrow R$ be given by

$$
U(x, y)= \begin{cases}y, & \text { if } x=0 \\ 0, & \text { if } 0<x \leq 1\end{cases}
$$

Then $U(x, y)$ is quasiconcave and upper semicontinuous in $x$ for each fixed $y$ and is quasiconvex, in fact convex, and lower semicontinuous in $y$ for each fixed $x$. The set of saddle points is $[0,1] \times\{0\}$. The best response maps are

$$
B R_{1}(y)=\arg \max _{x} U(x, y)= \begin{cases}{[0,1],} & \text { if } y=0 \\ 0, & \text { if } y>0\end{cases}
$$

and

$$
B R_{2}(x)=\arg \min _{y} U(x, y)= \begin{cases}0, & \text { if } x=0 \\ {[0,1],} & \text { if } x>0\end{cases}
$$

The first observation is that $B R_{2}$ is not upper semicontinuous at $x=0$.

The second observation is that

$$
\xi(t)=e^{-t}, \eta(t)=1-e^{-t}
$$

is a solution, in the Carathéodory and in the classical sense, to the best response dynamics

$$
\dot{\xi} \in B R_{1}(\eta)-\xi, \quad \dot{\eta} \in B R_{2}(\xi)-\eta .
$$

Indeed, at $x=1, y=0$ we have $B R_{1}(y)=[0,1], B R_{2}(x)=[0,1]$, while for $x \in$ $(0,1), y \in(0,1)$ we have $B R_{1}(y)=0, B R_{2}(x)=[0,1]$. Thus, for (3.1) and all $t \geq 0,0 \in B R_{1}(\eta(t)), 1 \in B R_{2}(\xi(t))$, and so (3.1), being the solution to $\dot{\xi}=-\xi, \dot{\eta}=$ $1-\eta$ from $(\xi(0), \eta(0))=(1,0)$, is a solution to the best response dynamics. Note that $(1,0)$ is a saddle point of $U$, while $\lim _{t \rightarrow \infty}(\xi(t), \eta(t))=(0,1)$ is not.

Our third observation is that using the augmented best response dynamics eliminates this particular troublesome solution. Indeed, we have the envelopes $U^{-}(x, y)=U(x, y), U^{+}(x, y)=(1-x) y$, and the augmented best response is

$$
B R_{1}^{-}(y)=\arg \max _{x} U^{-}(x, y)=\arg \max _{x} U(x, y)=B R_{1}(y)
$$

and

$$
B R_{2}^{+}(x)=\arg \min _{y} U^{+}(x, y)= \begin{cases}0, & \text { if } x<1 \\ {[0,1],} & \text { if } x=1\end{cases}
$$

Then, for $x \in(0,1], y \in(0,1]$, the augmented best response dynamics are $\dot{\xi}=$ $-\xi, \quad \dot{\eta}=-\eta$, and (3.1) is not a solution. Observe as well that we do not need to use the inf and sup convolutions of $U^{-}$and $U^{+}$, respectively, to fix the problem here.

One may obviously ask the question if the nonconvergence of the best response dynamics in this example is due to the choice of a discontinuous payoff function. Our next example will show that this is not the case.

Example 2. Let $U:[0,1] \times[-1,1] \rightarrow \mathbb{R}$ be given by

$$
U(x, y)=\max \{0, y-x\} \text {. }
$$


Then $U(x, y)$ is continuous, quasiconcave in $x$ for each fixed $y$ and quasiconvex, in fact convex in $y$ for each fixed $x$. The set of saddle points is $[0,1] \times[-1,0]$. The best response maps are

$$
\begin{gathered}
B R_{1}(y)=\arg \max _{x} U(x, y)=\left\{\begin{array}{ccc}
{[0,1]} & \text { if } y \in[-1,0], \\
0 & \text { if } y \in(0,1]
\end{array}\right. \\
B R_{2}(x)=\arg \min _{y} U(x, y)=[-1, x] .
\end{gathered}
$$

Then

$$
\begin{gathered}
\xi(t)= \begin{cases}\frac{3}{4} e^{-t} & \text { if } t \in\left[0,-\ln \frac{2}{3}\right), \\
\frac{1}{2}+\frac{1}{4}\left(t+\ln \frac{2}{3}\right) & \text { if } t \in\left[-\ln \frac{2}{3}, 1-\ln \frac{2}{3}\right],\end{cases} \\
\eta(t)= \begin{cases}\frac{1}{4} t & \text { if } t \in\left[0,-\frac{1}{2} \ln \frac{2}{3}\right), \\
-\frac{1}{8} \ln \frac{2}{3}-\frac{1}{4}\left(t+\frac{1}{2} \ln \frac{2}{3}\right) & \text { if } t \in\left[-\frac{1}{2} \ln \frac{2}{3},-\ln \frac{2}{3}\right), \\
0 & \text { if } t \in\left(-\ln \frac{2}{3}, 1-\ln \frac{2}{3}\right]\end{cases}
\end{gathered}
$$

is a solution, in the Carathéodory sense, to the best response dynamics. Indeed, for $x \in[1 / 2,3 / 4], y \in(0,1 / 4]$, we have

$$
B R_{1}(y)=0, \quad\left\{\frac{1}{4},-\frac{1}{4}\right\} \subset B R_{2}(x)
$$

and for such $x, y$,

$$
\dot{\xi}=-\xi, \quad \dot{\eta}=\frac{1}{4} \text { or } \dot{\eta}=-\frac{1}{4}
$$

are selections of the best response dynamics. For $x \in[1 / 2,3 / 4], y=0$, we have

$$
\frac{1}{4} \in B R_{1}(y), \quad 0 \in B R_{2}(x)
$$

and for such $x, y$,

$$
\dot{\xi}=\frac{1}{4}, \quad \dot{\eta}=0
$$

are selections of the best response dynamics. In simpler words, the solution of (3.2), (3.3) starts at $(3 / 4,0)$, moves according to $\dot{\xi}=-\xi, \dot{\eta}=1 / 4$ for some time; then according to $\dot{\xi}=-\xi, \dot{\eta}=-1 / 4$ until it returns to the $x$-axis; then according to $\dot{\xi}=1 / 4, \dot{\eta}=0$ until it returns to the initial point.

Now, the solution of (3.2), (3.3) starts at $(3 / 4,0)$ and returns to that point at $t=1-\ln \frac{2}{3}$. It can then be extended periodically to a solution of the best response dynamics that starts in the set of saddle points but does not converge to a saddle point. Indeed it cycles and does not converge at all.

Example 3. In this last example we illustrate the convergence of the best response dynamics for the concave-convex case. Take the function $U(x, y)=x y,(x, y) \in$ $[-1,1] \times[-1,1]$. The best response dynamics can be written explicitly as

$$
\dot{\xi}=-\xi+\frac{\eta}{|\eta|}, \quad \dot{\eta}=-\eta-\frac{\xi}{|\xi|}, \quad \tau>0,
$$

assuming we have the initial point $(\xi(0), \eta(0)) \neq(0,0)$. The function $V$ is given by

$$
V(x, y)=\max _{x} U(x, y)-\min _{y} U(x, y)=|x|+|y| .
$$


In Figure 1 on the left we plot the solutions of the best response dynamics as well as $v(\tau)=|\xi(\tau)|+|\eta(\tau)|$. On the right we indicate the convergence of $(\xi(\tau), \eta(\tau)) \rightarrow$ $(0,0)$ along the surface of the payoff function $U(x, y)=x y$.
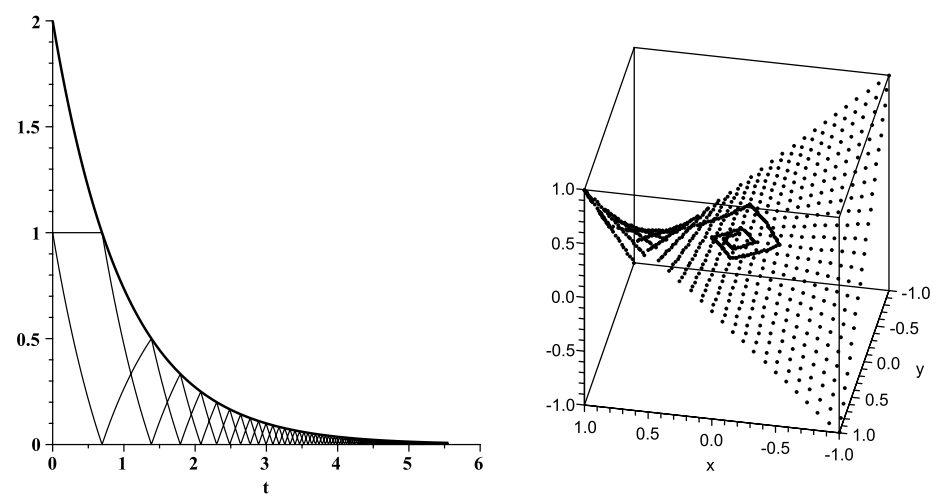

FiguRE $1 . V$ strictly decreases along trajectories

As indicated, the function $v(\tau)$, which is the highest function in the figure, strictly decreases along the trajectories, which converge to the unique saddle point $\left(x^{*}, y^{*}\right)=(0,0)$. The curves below $v(\tau)$ are $|\xi(\tau)|,|\eta(\tau)|$. Observe that they do not decrease.

\section{Best Response DyNAMiCs DON'T WORK FOR NASH EQUILIBRIA}

We construct a 3-player, nonzero sum game for which the best response dynamics do not converge to a Nash equilibrium for the game.

The three players each have the individual payoff functions

$$
\begin{aligned}
& u_{1}(x, y, z)=(x+3 y-2 z)^{2}, \\
& u_{2}(x, y, z)=(y-x-z)^{2}, \\
& u_{3}(x, y, z)=(z-2 x-y)^{2} .
\end{aligned}
$$

Observe that these functions are strictly convex. The players have a unique Nash equilibrium, $\left(x^{*}, y^{*}, z^{*}\right)=(0,0,0)$.

The best response dynamics are given by

$$
\begin{aligned}
\dot{x} & =-x-3 y+2 z, \\
\dot{y} & =x-y+z, \\
\dot{z} & =2 x+y-z .
\end{aligned}
$$

This is a homogeneous linear system which produces the eigenvalues $\lambda=-3, \pm i$.

The solution of this system with initial point $(x(0), y(0), z(0))=(1,1,1)$ is

$$
\begin{aligned}
& x(t)=\frac{2}{5} e^{-3 t}-\frac{4}{5} \sin t+\frac{3}{5} \cos t, \\
& y(t)=\cos t+\sin t \\
& z(t)=-\frac{2}{5} e^{-3 t}+\frac{4}{5} \sin t+\frac{7}{5} \cos t .
\end{aligned}
$$


It is easy to verify that $(x(t), y(t), z(t)), t \geq 0$, from any initial point in $\mathbb{R}^{3}$ converges to a closed space curve cycling about the origin. See Figure 2 ,

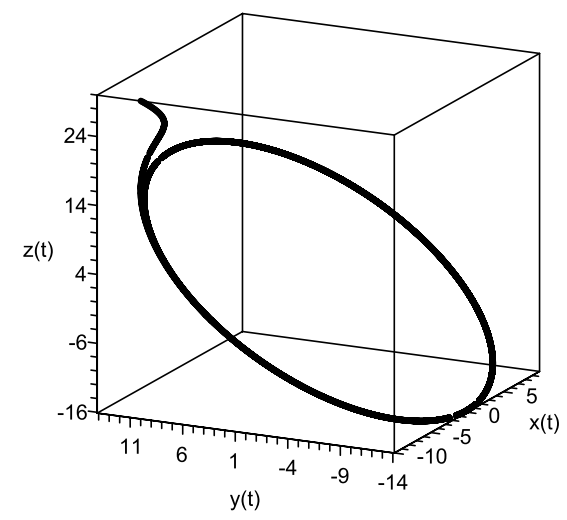

Figure 2. Convergence to a closed curve

Consequently, the best response dynamics do not converge to the Nash equilibrium.

Remark 4.1. In 1951 G.W. Brown [3] defined a discrete fictitious play process for the game $U$ by

$$
p_{n+1} \in B R_{1}\left(Q_{n}\right), \quad q_{n+1} \in B R_{2}\left(P_{n}\right)
$$

with initial values $p_{1}=P_{1} \in X, q_{1}=Q_{1} \in Y$, and for $n \geq 1$,

$$
P_{n}=\frac{1}{n} \sum_{k=1}^{n} p_{k}, \quad Q_{n}=\frac{1}{n} \sum_{k=1}^{n} q_{k} .
$$

In this way, as pointed out in [4, we get the discrete version of the BR dynamics:

$$
(n+1) P_{n+1}-n P_{n} \in B R_{1}\left(Q_{n}\right), \quad(n+1) Q_{n+1}-n Q_{n} \in B R_{2}\left(P_{n}\right)
$$

or

$$
P_{n+1}-P_{n} \in \frac{1}{n+1}\left[B R_{1}\left(Q_{n}\right)-P_{n}\right] \text { and } Q_{n+1}-Q_{n} \in \frac{1}{n+1}\left[B R_{2}\left(P_{n}\right)-Q_{n}\right] \text {. }
$$

A referee has kindly pointed out that in [6] Shapley exhibited a two-person nonzero sum game for which the fictitious play process, and hence the discrete BR dynamics, do not converge to a Nash equilibrium. The game was given by a version of rock-paper-scissors with the bimatrix

\begin{tabular}{|c|c|c|c|}
\hline & $A$ & $B$ & $C$ \\
\hline$a$ & 0,0 & 1,0 & 0,1 \\
\hline$b$ & 0,1 & 0,0 & 1,0 \\
\hline$c$ & 1,0 & 0,1 & 0,0 \\
\hline
\end{tabular}

Allowing mixed strategies, we have the strategy sets $X=Y=[0,1]^{3}$. There is a unique mixed Nash equilibrium given by $P=\left(\frac{1}{3}, \frac{1}{3}, \frac{1}{3}\right)=Q$; i.e., both players play 
each pure strategy with probability $\frac{1}{3}$. If the players start at $p_{1}=(1,0,0) \in X, q_{1}=$ $(0,1,0) \in Y$, it can be shown that the BR dynamics do not converge to the Nash equilibrium.

\section{REFERENCES}

1. F. Clarke, Y. Ledyaev, R. Stern, and P. Wolenski, Nonsmooth Analysis and Control Theory, Springer-Verlag, New York, 1998. MR1488695 (99a:49001)

2. I. Gilboa and A. Matsui, Social stability and equilibrium, Econometrica 59 (1991), 859-867. MR 1106514

3. G.W. Brown, Iterative solutions of games by fictitious play, Activity Analysis of Production and Allocation (T.C. Koopmans, ed.), Wiley, New York, 1951. MR0056265 (15:48e)

4. J. Hofbauer and S. Sorin, Best response dynamics for continuous zero-sum games, Discrete and Continuous Dynamical Systems Ser. B 6 (2006), no. 1, 215-224. MR2172204 (2007b:34027)

5. J.-P. Aubin and A. Cellina, Differential Inclusions, Springer-Verlag, Berlin, 1984. MR.755330 $(85 \mathrm{j}: 49010)$

6. L.S. Shapley, Some topics in two-person games, Advances in Game Theory (M. Drescher, L.S. Shapley, and A.W. Tucker, eds.), Princeton University Press, Princeton, N.J., 1964. MR0198990 (33:7140)

7. R.T. Rockafellar and R. Wets, Variational Analysis, Springer-Verlag, Berlin, 1998. MR 1491362 (98m:49001)

Department of Mathematics and Statistics, Loyola University Chicago, Chicago, ILLINOIS 60626

E-mail address: ebarron@luc.edu

Department of Mathematics and Statistics, Loyola University Chicago, Chicago, ILLINOIS 60626

E-mail address: rgoebel@luc.edu

Department of Mathematics and Statistics, Loyola University Chicago, Chicago, ILLINOIS 60626

E-mail address: rjensen@luc.edu 